\title{
Near-field Communication (NFC)
}

An Alternative to RFID in Libraries

Neeraj Kumar Singh

\section{ABSTRACT}

Libraries are the central agencies for the dissemination of knowledge. Every library aspires to provide maximum opportunities to its users and ensure optimum utilization of available resources. Hence, libraries have been seeking technological aids to improve their services. Near-field communication (NFC) is a type of radio-frequency technology that allows electronics devices-such as computers, mobile phones, tags, and others-to exchange information wirelessly across a small distance. The aim of this paper is to explore NFC technology and its applications in modern era. The paper will discuss potential use of NFC in the advancement of traditional library management system.

\section{INTRODUCTION}

Similar to other identification technologies such as radio-frequency identification (RFID), barcodes, and QR codes, near-field communication (NFC) is a short-range $(4-10 \mathrm{~cm})$ wireless communication technology. NFC is based on the existing 13.56 MHZ RFID contactless card standards which have been established for several years and are used for payment, ticketing, electronic passport, and access control among many other applications. Data rates range from 106 to 424 kilobits per second. A few NFC devices are already capable of supporting up to 848 kilobits per second which is now being considered for inclusion in the NFC Forum specifications. ${ }^{1}$

Compared to other wireless communication technologies NFC is designed for proximity or shortrange communication which provides a dedicated read zone and some inherent security. Its 13.56 MHz frequency places it within the ISM band, which is available worldwide. It is a bi-directional communication meaning that you can exchange data in both directions with a typical range of 4$10 \mathrm{~cm}$ depending on the antenna geometry and the output power. ${ }^{2} \mathrm{NFC}$ is convenient and fast: the action is automatically triggered when your phone comes within $10 \mathrm{~cm}$ near the NFC tag and you get instant access to the content on mobile, without a single click. ${ }^{3}$

RFID and NFC technologies are similar in that both use radio waves. Both RFID and NFC technologies exchange data within electronic devices in active mode as well as in passive mode. In the active mode, outgoing signals are basically those that actually come from the power source, whereas in case of passive mode the signals use the reflected energy they have received from the active signal. In RFID technology the radio waves can send information to receivers up to hundreds of meters away depending on the frequency of the band used by the tag. If provided with high amount of power, these signals can also be sent to extreme distances (e.g., in the case of airport radar). At large airports it typically controls traffic within a radius of 100 kilometers of the airport below an elevation of 25,000 feet. RFID is also used very often in tracking animals and vehicles.

Neeraj Kumar Singh (neerajkumar78ster@gmail.com), PhD, is Deputy Librarian, Panjab University, Chandigarh, India 
In contrast, items like passports and payment cards should not be capable of long-distance transmissions because of the threat of theft of personal information or funds. NFC is designed to meet this need. NFC tags are very small in size so as to fit on the inner side of devices and products such as inside luggage, purses and packs as well as from inside wallets and clothing and can be tracked. NFC technology has added security features that make it much more secure than the previously popular RFID equivalent and it is difficult to steal information stored in it. NFC has short range of work area compared to other wireless technologies, so it can be widely used for payments, ticketing and service admittance and thus has proved to be a safer technology. It is because of this security feature that this technology is used in cellular phones to turn them into a wallet. $^{4}$

Both RFID and NFC wireless technologies can operate in active and passive communication modes to exchange data within electronic devices. The main differences between NFC and RFID are:

- Though both RFID and NFC use radio frequencies for communication, NFC can be said to be an extension of the RFID technology. The RFID technology has been in use for more than a decade, but NFC has emerged on the scene recently.

- RFID has a wider range whereas NFC has limited communication and operates only at close proximity. NFC typically has a range of a few centimeters.

- $\quad$ RFID can function in many frequencies and many standards are being used, but NFC requires a fixed frequency of $13.56 \mathrm{MHz}$, and some other fixed technical specifications to function properly.

- RFID technology can be used for such applications as item tracking, automated toll collecting on roads, vehicle movement, etc., that require wide area signals. NFC is appropriate for applications that carry data that needs to be kept secure like mobile payments, access controls, etc., that carry sensitive information.

- RFID operates over long distances while exchanging data wirelessly so it is not secure for the applications that store personalized data. RFID using items susceptible to various fraud attacks such as data corruption. NFC's short working range considerably reduces this risk of data theft, eavesdropping, and "man in the middle" attacks.

- NFC has the capability to communicate both ways and thus is suitable to be used for advanced interactions such as card emulation and peer-to-peer sharing.

- A number of RFID tags can be scanned simultaneously, while only a single NFC tag can be scanned at a time.

\section{HOW NFC WORKS}

The extended functionality of a traditional RFID system has led to the NFC Forum. The NFC Forum has defined three operating modes for NFC devices: tag reader/writer mode; peer-to-peer mode, and card emulation mode (see figure 1). The NFC Forum technical specifications for the different operating modes are based on the ISO/IEC 18092 NFC IP-1, JIS X 6319-4, and ISO/IEC 14443. These specifications must be used to derive the full benefit from the capabilities of NFC technology. Contactless smart card standards are referred to as NFC-A, NFC-B, and NFC-F in NFC Forum specifications. $^{5}$ 


\section{NFC Devices Operate in 3 Modes}

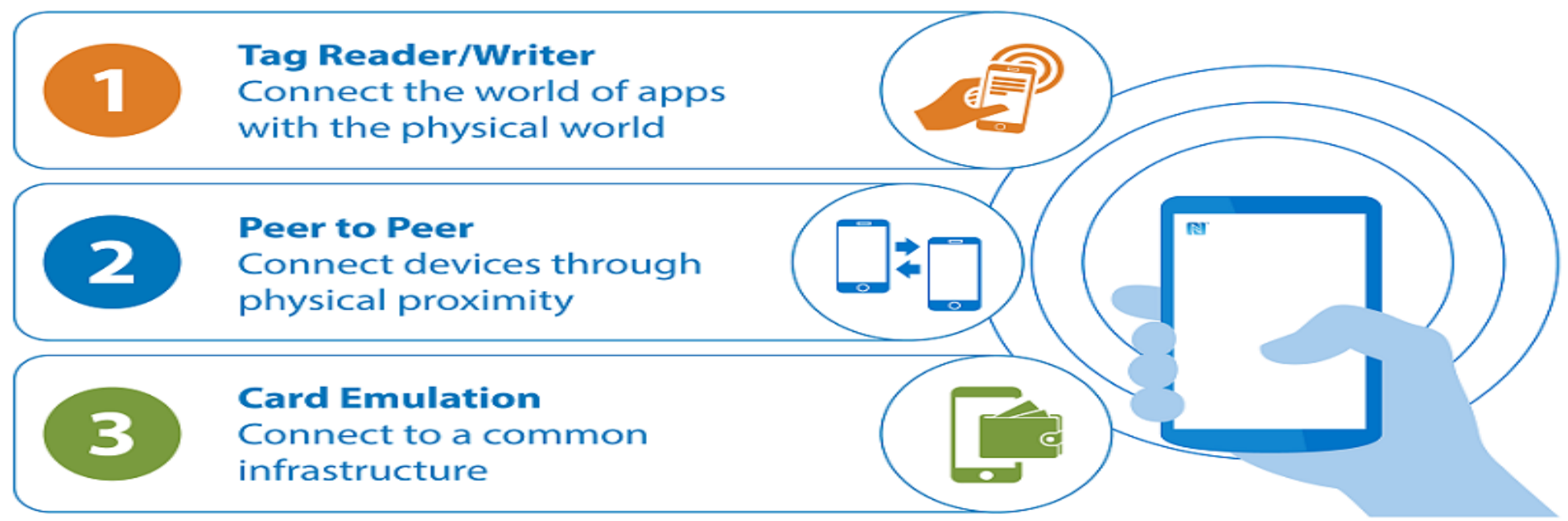

Figure 1. NFC Operation Modes 6

\section{Reader/Writer Mode}

In reader/writer mode (see figure 2), an NFC-enabled device is capable of reading NFC Forummandated tag types, such as a tag embedded in an NFC smart poster. This mode allows NFCenabled devices to read the information that is stored on NFC tags embedded in smart posters and displays. Since these tags are relatively inexpensive, they provide a great marketing tool for companies.

\section{Initiator}

Target

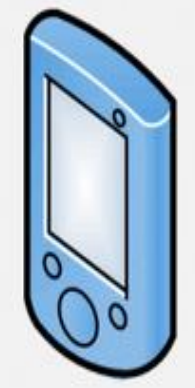

Smartphone (Active Device)

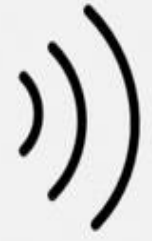

Reader/Writer Mode ASK Modulation

Load Modulation
13.56 MHz RF

NFC Tag

(Passive Device)

Figure 2. Reader Mode7

The reader/writer mode on the radio frequency interface is compliant with the NFC-A, NFC-B, and NFC-F schemes. Examples of its use include reading timetables, tapping for special offers, and updating frequent flyer points, etc. ${ }^{8}$ 


\section{Peer-to-Peer Mode}

In peer-to-peer mode (see figure 3), both devices must be NFC-enabled in order for them to communicate with each other to exchange information and to share files. The users of NFCenabled devices can thus quickly share information and other files with a touch. As an example, users can exchange data such as digital photos or virtual business cards via Bluetooth or WiFi.
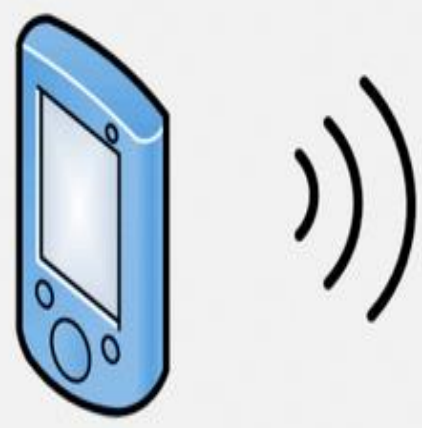

Smartphone (Active Device)

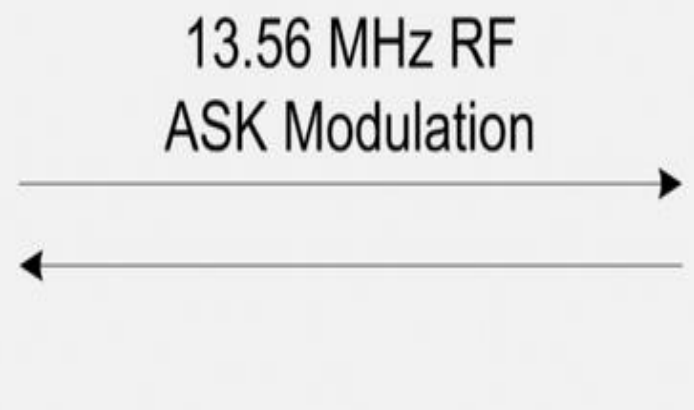

Peer-to-Peer Mode

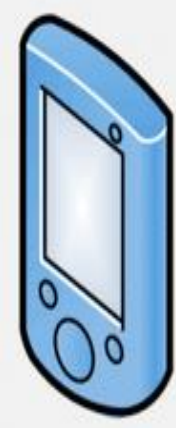

Smartphone

(Active Device)

Figure 3. Peer-to-Peer Mode ${ }^{9}$

Peer-to-peer mode is based on the NFC Forum's Logical Link Control Protocol Specification and is standardized on the ISO/IEC 18092 standard.

\section{Card-Emulation Mode}

In card-emulation mode (see figure 4), an NFC device behaves like a contactless smart card so that users can perform transactions such as purchases, ticketing, and transit access control with just a touch. An NFC device may have the ability to emulate more than one card. In card-emulation mode, an NFC-enabled device communicates with an external reader much like a traditional contactless smart card. This allows contact less payments and ticketing by NFC-enabled devices without changing the existing infrastructure. 


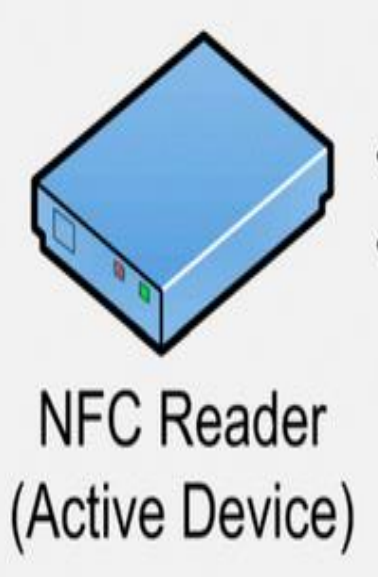

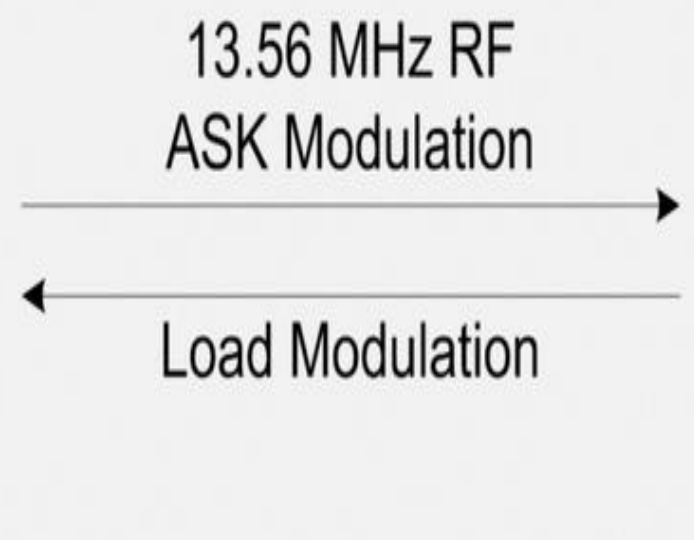

Card Emulation Mode

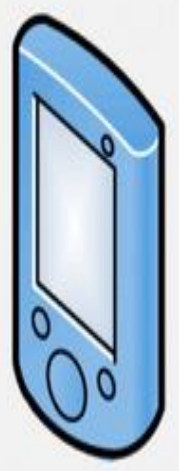

Smartphone

(Passive Device)

Figure 4. Card-Emulation Mode

By adding NFC to a contactless infrastructure one can enable two-way communications. In the air transport sector, this could simplify many operations such as updating seat information while boarding or adding frequent flyer points while making a payment. ${ }^{10}$

\section{NFC STANDARDS AND SPECIFICATIONS}

The NFC specifications are defined by an industry organization called the NFC Forum, which has nearly 200 member companies. The NFC Forum was formed in 2004 with the objective of advancing the use of NFC technology. This was achieved by educating the market about NFC technology and developing specifications to ensure interoperability among devices and services. The NFC Forum members are working together in task forces and working groups. As noted earlier, NFC technology is based on existing 13.56 MHZ RFID standards and includes several protocols such as ISO 14443 type A and type B, and JIS X 6319-4 (which is also a Japanese Industrial standard known as Sony FeliCa). The ISO 15693 standard, an additional $13.56 \mathrm{MHZ}$ protocol established in the market, is being integrated into the NFC specification by an NFC Forum task force. Smartphones in the market are already supporting the ISO 15693 protocol. ${ }^{11}$ These NFC specifications and especially the specifications for the extended NFC functionalities are again standardized by the international standard organizations like ISO/IEC ECMA and ETSI. ${ }^{12}$

Initially the RFID standards i.e. ISO/IEC 14443 A, ISO/IEC 14443 B and JIS X6319-4 were also pronounced as NFC Standards by Different companies working in the field such as NXP, Infineon, and Sony. The first ever NFC standard was ECMA 340, based on the Air Interface of ISO/IEC 14443A and JIS X6319-4. ECMA 340 adapted the ISO/IEC standard 18092. At the same time, major credit card companies like Europay, Mastercard, and Visa introduced the EMVCo payment standard, which is based on ISO/IEC 14443 A and ISO/IEC $14443 \mathrm{~B}$. These groups harmonised the over-the-air interfaces within the NFC Forum. They are named NFC-A (ISO/IEC 14443 A based), NFC-B (ISO/IEC 14443 B based), and NFC-F (FeliCa based). ${ }^{13}$ 


\section{NFC Tags}

An NFC tag is a small microchip embedded in a sticker or wristband that can be read by the mobile devices that are within range. Information regarding the item is stored in these microchips. ${ }^{14} \mathrm{An}$ NFC tag has the capability to send the information stored on it to NFC enabled mobile phones. NFC tags can also perform various actions, such as changing the settings of handsets or even launch a website. $^{15}$

Tag memory capacity varies by the type of tag. For example, a tag may store a phone number or a URL. ${ }^{16}$ The most common use of the NFC tag function on an object is mobile wallet payment processing, where the user swipes or flicks a mobile phone on a NFC tag to make payment. Google's version of this system is Google Wallet. ${ }^{17}$

\begin{tabular}{|c|c|c|c|c|c|}
\hline & \multicolumn{5}{|c|}{ NFC Forum Platform } \\
\hline & $\begin{array}{c}\text { Type } 1 \text { Tag } \\
\text { NFC-A Compliant }\end{array}$ & $\begin{array}{c}\text { Type } 2 \text { Tag } \\
\text { NFC-A Compliant }\end{array}$ & $\begin{array}{c}\text { Type } 3 \text { Tag } \\
\text { NFC-F Compliant }\end{array}$ & $\begin{array}{c}\text { Type } 4 \text { Tag } \\
\text { NFC-A, NFC-B Compliant }\end{array}$ & $\begin{array}{c}\text { Type } 5 \text { Tag } \\
\text { NFC-V Compliant }\end{array}$ \\
\hline $\begin{array}{l}\text { Compatible } \\
\text { Products }\end{array}$ & Broadcom Topaz & $\begin{array}{l}\text { NXP MIFARE Ultralight/ } \\
\text { NXP MIFARE Ultralight C } \\
\text { NXP NTAG } 21 \mathrm{~s}(\mathrm{~F}) \\
\text { NXP NTAG } 12 \mathrm{C}\end{array}$ & Sony FeliCa & $\begin{array}{c}\text { NXP DESFire/ } \\
\text { NXP Smart MX-JCOP } \\
\text { (MIFARE DESFire } \\
\text { implementation)/ } \\
\text { ST Microelectronics } \\
\end{array}$ & $\begin{array}{c}\text { NXP ICODE SLI }(\mathrm{x}) / \\
\text { Texas Instruments Tag -lt } \\
\text { HF-I/ } \\
\text { EM423X/ } \\
\text { ST Microelectronics } \\
\end{array}$ \\
\hline Memory Size & 454 Bytes & $\begin{array}{c}48 / 128 / 144 \text { / } 504 / 888 \\
\text { / } 1904 \text { Bytes }\end{array}$ & 1, 4, 9 KBytes & $\begin{array}{l}2 / 4 / 8 \text { Kbytes } \\
\text { up to } 144 \text { Kbytes / } \\
106 \text { Kbytes }\end{array}$ & $\begin{array}{c}32 / 112 / 128 / 160 / \\
256 \text { Bytes }\end{array}$ \\
\hline Unit Price & Low & Low & High & Medium / High & Low / Medium \\
\hline Data Access & Read/Write & $\begin{array}{l}\text { Read/Write or } \\
\text { Read-only }\end{array}$ & $\begin{array}{l}\text { Read/Write or } \\
\text { Read-only }\end{array}$ & $\begin{array}{l}\text { ReadWrite or } \\
\text { Read-only }\end{array}$ & Read/Write \\
\hline Active Content* & & & & $x / 0$ & \\
\hline $\begin{array}{l}\text { Operation } \\
\text { Specification }\end{array}$ & ISO/IEC 14443-3 A & ISO/IEC 14443-3 A & HS 6319.4 & ISO/IEC 14443.4 A/B & ISO/IEC 15693 \\
\hline
\end{tabular}

Figure 5. A Quick Overview of the Tag Types ${ }^{\mathbf{1 8}}$

\section{Applications of NFC}

Since it emerged as a standard technology in 2003, NFC technology has been implemented across multiple platforms in various ways. The primary driving force behind NFC is its application in the commercial sector in which the implementation of the technology focuses on such areas as sales and marketing. There are also emerging many new and interesting applications in various other fields of education and healthcare. All of these may impact libraries, librarians, and library users, either by prompting adaptations to existing collections and services or inspiring innovation in our profession. ${ }^{19}$

- Mobile payment: Customers with NFC-enabled smartphones can link with their bank accounts and are able to pay by simply tapping phones to an NFC-enabled point-of-sale. ${ }^{20}$ 
- Access and authentication: "keyless access" to restricted areas, cars, and other vehicles. One can imagine other potential uses of NFC in the future with the devices in the home being controlled by it. ${ }^{21}$

- Transportation and ticketing: NFC-enabled phones can connect with an NFC-enabled kiosk to download a ticket, or the ticket can be sent directly to an NFC-enabled phone over the air (OTA). The phone can then tap a reader to redeem that ticket and gain access. ${ }^{22}$

- Mobile marketing: NFC tags they can be embedded into the indoor and outdoor signage. Upon tapping their smartphone on an NFC-enabled smart poster, the customer can read a consumer review, visit a website, or even view a movie trailer.

- Healthcare: NFC medical cards and bracelet tags can store relevant, up-to-date patient information like health history, allergies, infectious diseases, etc.

- Gaming: NFC technology is the bridge between physical and digital games. Players can tap each other's phones together and earn extra points or receive access to a new level, or get clues, by using NFC application. ${ }^{23}$

- Inventory tracking, smart packaging, and shelf labels: NFC-tagged objects could provide a wide variety of information in different use environments. NFC-enabled smartphones can be used to tap the tags to access book reviews and information about the book's author and recommend the book to other readers. Users could check out a book or add it to a wish list to check out at a later date. Indeed, with NFC, library records and metadata could theoretically be stored on and retrieved from library physical holdings themselves, allowing a patron to tap a book or resource borrowed from the library to recall its title, author, and due date. ${ }^{24}$

\section{APPLICATIONS OF NFC IN LIBRARIES: INTRODUCING THE SMART LIBRARY}

Some libraries are beginning to use NFC technology as an alternative to RFID. Yusof et al. proposed a newly developed application called the Smart Library, or "S-Library," that has adopted the NFC technology. ${ }^{25}$ In the S-Library, library users can perform many library transactions just by using their mobile smartphones with integrated NFC technology. The users of S-Library are required to download and install an app in their compatible mobile phone. This app provides the user relevant and easy to use library functionality such as searching, borrowing, returning, and viewing their transaction records. In this S-Library model the app is integrated with the library management software. The S-library app needs to be installed on the mobile device, and the mobile device requires an internet connection that will connect it to the LMS.

The S-Library provides five major functionalities to the user: scan, search, borrow, return, and transaction history. In the scanning function, users can access the information of a book by simply touching their mobile phone to the NFC tag on the book. As soon as the phone touches the book, information regarding its title, author, contents, synopsis, etc. will automatically be displayed on the screen of the mobile device. Users can search for books by entering keywords such as book title, author name, year, etc. Through the borrowing function the app allows users to check out books of interest. The user just needs to touch their mobile phone to the NFC-tagged book to borrow it. The transaction is automatically stored to the LMS database. Similar to the borrowing process is the returning process. The user is required to select the return function on the menu and touch the mobile device to the book, and the returning transaction will be automatically performed and stored in the LMS database. However, it should be ensured that the book is physically returned to the library by returning the book through the NFC-enabled book drop 
system of the library and only then transaction should be updated in the LMS. The user can check the due date for the current transaction as well as his transaction history. The function of transaction history allows the user to view the list of books that have been borrowed from time to time and their status. ${ }^{26}$

Data transmission for NFC technology can be up to 848 kilobits/second whereas the data transmission rate with RFID technology is 484 kilobits/second. Taking advantage of this high data rate, the response time for S-Library is also very fast. This is a huge improvement over RFID technology and especially over barcode technology where data transmission rate is variable and inconsistent and dependent upon the quality of the barcodes. The second key advantage of SLibrary is that the time taken to read a tag (the communication time between a reader and an NFC enabled device) is very fast. The third advantage of NFC is its usability in comparison to the other two technologies. NFC technology is human-centric because it is intuitive and fast and the user is able to use it anywhere, anytime using their mobile phones. In RFID and barcode technology usability is item centric as person has to go to the specific device located in the library. ${ }^{27}$

Most of the shortcomings of RFID and barcode technology have been overcome by the S-Library. With barcode technology, the quality of barcodes, printing clarity, print contrast ratio, and also the low level of security were all challenges. RFID technology had many drawbacks such as lack of common RFID standards, security vulnerability, reader and tag collision that happens when multiple tags are energized by the RFID tag reader simultaneously and they reflect their respective signals back to the reader at the same time. Because NFC is touch based, it has presented a viable alternative tool for library users to overcome these weaknesses of the older technology.

Yosof et al. found many advantages to S-Library: faster book borrowing; saved time of the user as well as the library staff; the connection can be initialised in less than a second; no configuration on the mobile device is required; and higher usability ratings and security. ${ }^{28}$ However, there are also some limitations of S-Library. First, device compatibility is an issue, because S-Library presently supports only the Android platform. Second, as the S-Library application only supports up to a 10centimeter range, coverage is an issue.

\section{Mobile Payments}

NFC technology can be used for several library functions such as making payments, paying library fines, purchasing tickets to library events, or donating to library. Users may also be able to use their digital wallet to pay for photocopying, printing, scanning, etc. Keeping the requirements of the NFC technology in the future libraries have to enquire about the possibility of adding NFC payment capabilities into the existing hardware and also while purchasing new machines. Already, Bibliotheca's Smartserv 1000 self-serve kiosk, introduced in September 2013, includes NFC as a payment option. In the future other library automation companies for NFC integration would also be worth monitoring. ${ }^{29}$

\section{Library Access and Authentication}

NFC-enabled devices can be used to accessing the library and authenticate users. These capabilities suggest that NFC technology may play an important role in the next generation of identity management systems. Of particular interest in this context are several applications of NFC in two-factor authentication, which generally combines a traditional password or other digital credential with a physical, NFC-enabled component as well. For example, an authentication system 
could require the user to type in a fixed password in addition to tapping an NFC-enabled phone, identity card, or ring to the device they are logging in to. IBM has demonstrated a two-factor authentication method for mobile payment in which a user first types in a password and then taps an NFC-enabled credit card, issued by their bank, to their NFC-enabled smartphone.

Libraries could investigate similar access and authentication applications for NFC, both for internal use (staff badges and keys) as well as for public services. Particularly if NFC mobile payment finally gains consumer attraction, library patrons may begin to expect that they can use their NFC-enabled mobile devices to replace not just their credit cards but also their library cards. Already, D-Tech's RFID AIR Self Check unit allows library patrons to log into their user accounts by tapping their NFC-enabled phone to the kiosk. The patron then uses the kiosk's RFID reader to check out their library materials and receives a receipt via email or SMS. Beyond its application in circulation, NFC authentication can be applied to streamline access to other services and resources of the library. ${ }^{30}$ NFC-enabled devices could be used to make reservation of library spaces, classrooms, auditoriums or community halls, digital media labs, meeting rooms, etc. Library users could use NFC authentication to be able to access digital library resources, such as databases, e-journals, e-books collections, and other digital collections. NFC might allow libraries of all kinds to provide more convenient access and authentication options to users, though privacy and security considerations would certainly need to be addressed. NFC access and authentication will certainly have an impact on academic libraries. At universities where NFC access systems are deployed, student identification cards can be replaced with NFC-enabled mobile phones for afterhours services such as library building entry, WiFi access, and printing, copying, and scanning services. The inconvenience of multiple logins can be eliminated. However, the libraries will have to take the responsibility of protecting student information and library resources with added security. ${ }^{31}$

\section{Promotion of Library Services}

Librarians can borrow ideas from commercial implementations of NFC-based marketing to enhance promotions for library resources, services, and events. As a first step, as Kane and Schneidewind suggested, NFC tags can complement several promotional uses of QR codes that have already been piloted or implemented in libraries. ${ }^{32}$ For promotional use, libraries can easily embed NFC tags in their new book displays that can be linked to the bestseller list or current acquisitions lists in the library catalog or digital collections. Similarly, if the reference book collection is tagged with NFC tags, it could be linked to the relevant digital collections of databases or e-books. NFC tags can be placed on library building doors or on library promotional material by which information such as library hours, opening days, schedule of events, membership rules, or floor plans for the building could be shared. As an example, at the Renison University College Library in Ontario, Canada, visitors can tap an NFC-enabled "library smartcard" to retrieve a digital brochure of library services in a variety of formats, including PDF, EPUB, and MP3. ${ }^{33}$

To promote outreach programs and events instead of merely sharing links the libraries can take advantage of NFC's interactive capabilities. As an example, libraries could use NFC tags on their event posters so that the users that can scan them and register for an event, save the event to their personal calendar, join the Friends of the Library program, or even download a library app. To send a text message to a librarian the users can tap the smart poster promoting a virtual reference service. NFC-enabled promotional materials can engage users with library content even when they are outside of the library building itself. A brilliantly creative example was created by the Field 
Museum of Chicago. It used NFC-enabled outdoor smart posters throughout the city to promote an exhibit of the 1893 World's Fair. The event posters depicted a personage from 1893 that invited the viewer to "See What They Saw." Users could tap their NFC-enabled mobile device to the smart poster (or read a QR code) to download an app from the Field Museum that included $360^{\circ}$ images of the fair as well as videos highlighting items in the exhibition. ${ }^{34}$

\section{Inventory Control}

The smart packaging use case brings forward a very important question for libraries that use RFID for inventory control. First, can existing RFID tags and infrastructure be leveraged to provide additional services to patrons with NFC-enabled mobile devices? The concept is not new; Walsh envisioned using library RFID tags to store book recommendations or other digital information, which users could then access with a conveniently located RFID reader. ${ }^{35}$ What NFC brings to Walsh's vision is that a dedicated RFID reader may no longer be necessary; a patron could use their own NFC-enabled smartphone to read a tag rather than taking it to a special location to be read. Indeed, with NFC, library records and metadata could theoretically be stored on and retrieved from library physical holdings themselves, allowing a patron to tap a book or resource borrowed from the library to recall its title, author, and due date. An exciting and immediate use for NFC in libraries is for self-checkout: a patron can browse the stacks and could tap an NFCtagged book with their NFC-enabled phone to check it out without visiting the circulation desk or waiting in line. ${ }^{36}$

\section{Smart Packaging}

A sector close to librarians' hearts is publishing and several publishers have started testing smart packaging for books, using embedded NFC tags to share additional content with readers such as book reviews, reading lists, etc. With digital extras, the concept of smart packaging has significant implications for libraries as a new opportunity to connect physical collections (i.e., from books to digital media). One can envision in the future that when a user taps an NFC-enabled library book they shall get access to relevant digital information (such as bibliographic information) in a variety of citation formats, editorial reviews, the author's biography, a projected rating for the book, and links to other similar information.

\section{Borrowing and Returning Books}

One of a library's key functions is circulating physical books from the library's collections. Due to the low cost of barcode technology, many libraries around the world are using it for circulation management. However, barcode technology has several constraints: it requires a line-of-sight to the barcode, it does not provide security of library collection, it does not offer any benefit for collection management, and it is becoming challenging for libraries to satisfy the increasing demands of their users, for example, reservation of books issued out, checking their transaction history, etc. This leads to the need to implement a new technology to improve the library circulation management, inventory, and security of library collections. Librarians are known as early adopters of technology and have started using RFID to provide circulation services in a more effective and efficient manner, for security of library collections, and to satisfy the increasing demands of the users, for example putting tags in books allows them to issue multiple books together by placing stack of books near a reader. 


\section{RECOMMENDATIONS}

According to McHugh and Yarmey, the implementation of NFC has been slow and unsteady and they do not foresee an immediate implementation in libraries. ${ }^{37}$ However, they recommend that librarians learn and prepare for NFC. They recommend, for example, that librarians:

- follow the progress of research and scholarship on NFC and commercial progress of NFC technology to better anticipate its adoption in your community;

- experiment with NFC technology and develop prototype applications for NFC use in the library;

- offer an informational workshop on NFC for users and library colleagues;

- enquire from the RFID vendor about tag compatibility with NFC and rewriting the tags;

- monitor the progress of security and privacy aspects of NFC technology and educate the users about these issues; develop or update your library security policy;

- allow patrons to "opt-in" to any NFC services at your library, providing other modes of communication where possible;

- develop and share best practices for NFC implementations; and

- support research on NFC in libraries via planning grants, research forums, and conference sessions.

\section{CONCLUSIONS}

Beyond the potential benefits of NFC, librarians should also be aware of and prepared for privacy and security concerns that accompany the technology. User privacy is of the utmost concern. NFC involves users' mobile devices generating, collecting, storing, and sharing a significant amount of personal data. Several of these functions, particularly mobile payment, necessitate the exchange of highly confidential data, including but not limited to a user's financial accounts, purchase history, etc. Spam may also be a concern; sending unwanted content (e.g., advertisements, coupons, or adware) to users' mobile devices without their consent. Librarians should also use special caution when considering the implementation of NFC for library promotions or services. Security is a significant concern and an active area of research, as many NFC implementations involve the exchange of sensitive financial or otherwise personal data. An important concept in NFC security, particularly in the context of mobile payment, is the idea of a tamper-proof "secure element" as a basic protection for sensitive or confidential data such as account information and credentials for authentication. ${ }^{38}$

Outside of continued standardization, the most effective measures for protecting NFC data transmissions are data encryption and the establishment of a secure channel between the sending and receiving devices (e.g., using a key agreement protocol and/or via SSL). For security concerns, as with privacy concerns, librarians have a crucial role to play in user education. There are important steps that individual users can and should take to protect their devices - e.g., setting a lock code for their device, knowing how to remotely wipe a stolen phone, and installing and regularly updating antivirus software. However, many users are unaware of the vulnerability of their mobile devices and often fail to enact even basic protections.

By empowering objects and people to communicate with each other at a different level and establish a "touch to share" paradigm, NFC technology has the potential to transform the 
information environment surrounding our libraries and fundamentally alter the ways in which the library patrons interact with information.

\section{ENDNOTES}

${ }^{1}$ Doaa Abdel-Gaber and Abdel-Aleem Ali, "Near-Field Communication Technology and Its Impact in Smart University and Digital Library: Comprehensive Study," Journal of Library and Information Sciences, 3, no. 2 (December 2015): 43-77, https://doi.org/10.15640/jlis.v3n2a4.

2 "NFC Technology Discover what NFC is, and How to Use it," accessed March 17, 2019, https://www.unitag.io/nfc/what-is-nfc.

${ }^{3}$ Apuroop Kalapala, "Analysis of Near Field Communication (NFC) and other Short Range Mobile Communication Technologies" (Project Report, Indian Institute of Technology, Roorkee, 2013), accessed March 19, 2019, https://idrbt.ac.in/assets/alumni/PT-

2013/Apuroop $\% 20$ Kalapala_Analysis $\% 20$ of $\% 20$ Near $\% 20$ Field $\% 20$ Communication $\% 20$ (NFC) $\% 20$ and $\% 20$ other $\% 20$ short $\% 20$ range $\% 20$ mobile\%20communication\%20technologies_2013. pdf.

${ }^{4}$ Ed, “Near Field Communication vs Radio Frequency Identification," accessed March 10, 2019, http://www.nfcnearfieldcommunication.org/radio-frequency.html.

5 "What It Does," NFC Forum, accessed March 12, 2019, https://nfc-forum.org/what-is-nfc/whatit-does.

${ }^{6}$ José Bravo et al., “m-Health: Lessons Learned by m-Experiences,” Sensors 18, 1569 (2018): 1-27. $10.3390 / \mathrm{s} 18051569$.

7 Vedat Coskun, Busra Ozdenizci, and Kerem Ok, "The Survey on Near Field Communication," Sensors 15, no. 6 (2015): 13348-405, https://doi.org/10.3390/s150613348.

${ }^{8}$ Coskun, Ozdenizci, and Ok, “The Survey on Near Field Communications,” 13352.

${ }^{9}$ Coskun, Ozenizci, and Ok, "The Survey on Near Field Communication."

10 “How NFC Works?," CNRFID, accessed January 12, 2019, http://www.centrenationalrfid.com/how-nfc-works-article-133-gb-ruid-202.html.

${ }^{11}$ Coskun, Ozdenizci, and Ok, “The Survey on Near Field Communication,” 13352.

${ }^{12}$ C. Ruth, "NFC Forum Calls for Breakthrough Solutions for Annual Competition," accessed March 21, 2019, https://nfc-forum.org/newsroom/nfc-forum-calls-for-breakthrough-solutions-forannual-competition/.

${ }^{13}$ M. Roland, "Near Field Communication (NFC) Technology and Measurements," accessed May 12, 2019, https://cdn.rohdeschwarz.com/pws/dl_downloads/dl_application/application_notes/1ma182 /1MA182_5E_NFC_WHITE_PAPER.pdf.

${ }^{14}$ Roland, "Near Field Communication (NFC) Technology and Measurements." 
15 “What is a Near Field Communication Tag (NFC Tag)?," Techopedia, accessed May 27, 2019, https://www.techopedia.com/definition/28812/near-field-communication-tag-nfc-tag.

16 "What is Meant by the NFC Tag?," Quora, accessed July 12, 2019, https://www.quora.com/What-is-meant-by-the-NFC-tag.

${ }^{17}$ S. Profis, "Everything You Need to Know About NFC and Mobile Payments," accessed June 27, 2019, https://www.cnet.com/how-to/how-nfc-works-and-mobile-payments/.

18 "The 5 NFC Tag Types," accessed March 24, 2019, https://www.dummies.com/consumerelectronics/5-nfc-tag-types/.

${ }^{19}$ Abdel-Gaber and Ali, "Near-Field Communication Technology and Its Impact in Smart University and Digital Library," 64-71.

${ }^{20}$ Iviane Ramos de Luna et al., "NFC Technology Acceptance for Mobile Payments: A Brazilian Perspective," Review of Business Management 19, no. 63 (2017): 82-103, https://doi.org/10.7819/rbgn.v0i0.2315.

${ }^{21}$ Rajiv, "Applications and Future of Near Field Communication," accessed March 14, 2019, https://www.rfpage.com/applications-near-field-communication-future/.

22 “NFC in Public Transport," NFC Forum, accessed April 12, 2019, http://www.smartticketing.org/downloads/papers/NFC_in_Public_Transport.pdf.

23 "Gaming Applications with RFID and NFC Technology," SmartTech, accessed May 14, 2019, https://www.smarttec.com/en/applications/gaming.

${ }^{24}$ Sheli McHugh and Kristen Yarmey, "Near Field Communication: Recent Developments and Library Implications," Synthesis Lectures on Emerging Trends in Librarianship 1, no. 1 (March 2014), 1-93.

${ }^{25}$ M.K. Yusof et al., "Adoption of Near Field Communication in S-Library Application for Information Science," New Library World 116, no. 11/12 (2015): 728-47, https://doi.org/10.1108/nlw-02-2015-0014.

${ }^{26}$ Yusof et al., "Adoption of Near Field Communication," 734-36.

${ }^{27}$ Yusof et al., "Adoption of Near Field Communication," 744.

${ }^{28}$ Yusof et al., "Adoption of Near Field Communication," 745.

${ }^{29}$ Abdel-Gaber and Ali, "Near-Field Communication Technology and Its Impact in Smart University and Digital Library," 64.

${ }^{30}$ McHugh and Yarmey, "Near Field Communication," 27.

${ }^{31}$ McHugh and Yarmey, "Near Field Communication," 734. 
32 Danielle Kane and Jeff Schneidewind, "QR Codes as Finding Aides: Linking Electronic and Print Library Resources," Public Services Quarterly 7, no. 3-4 (2011): 111-24, https://doi.org/10.1080/15228959.2011.623599.

${ }^{33}$ McHugh and Yarmey, "Near Field Communication," 31.

${ }^{34}$ McHugh, and Yarmey, "Near Field Communication," 31.

${ }^{35}$ Andrew Walsh, "Blurring the Boundaries between our Physical and Electronic Libraries: Location-Aware Technologies, QR Codes and RFID Tags," The Electronic Library 29, no. 4 (2011): 429-37, https://doi.org/10.1108/02640471111156713.

${ }^{36}$ Projes Roy and Shailendra Kumar, "Application of RFID in Shaheed Rajguru College of Applied Sciences for Women Library, University of Delhi, India: Challenges and Future Prospects," Qualitative and Quantitative Methods in Libraries 5, no. 1 (2016): 117-130, http://www.qqmljournal.net/index.php/qqml/article/view/310.

${ }^{37}$ McHugh, and Yarmey, “Near Field Communication,” 61-2.

${ }^{38}$ Garima Jain and Sanjeet Dahiya, "NFC: Advantages, Limits and Future Scope," International Journal on Cybernetics \& Informatics 4, no. 4 (2015): 1-12, https://doi.org/10.5121/ijci.2015.4401. 